\title{
基于对抗投影学习的跨模态哈希检索
}

\author{
曾超 ${ }^{1)}$, 白琮 ${ }^{1}{ }^{*}$, 马青 2$)$, 陈胜勇 ${ }^{3)}$ \\ ${ }^{1)}$ (浙江工业大学计算机科学与技术学院 杭州 310023) \\ 2) (浙江工业大学理学院 杭州 310023) \\ ${ }^{3)}$ (天津理工大学计算机科学与工程学院 天津 300384) \\ (congbai@zjut.edu.cn)
}

\begin{abstract}
摘 要: 跨模态哈希检索以其较高的检索效率和较低的存储成本, 在跨模态检索领域受到了广泛的关注. 现有的跨 模态哈希大多直接从多模态数据中学习哈希码, 不能充分利用数据的语义信息, 因此无法保证数据低维特征在模态 间的分布一致性，解决这个问题的关键之一是要准确地度量多模态数据之间的相似度. 为此，提出一种基于对抗投 影学习的哈希(adversarial projection learning based Hashing for cross-modal retrieval, APLH)方法用于跨模态检索. 利用对 抗训练学习来自不同模态的低维特征, 并保证低维特征在模态间的分布一致性. 在此基础上, 利用跨模态投影匹配约 束(cross-modal projection matching, CMPM)，最小化特征投影匹配分布和标签投影匹配分布之间的 KL(Kullback-Leibler) 散度, 利用标签信息使数据低维特征之间的相似度结构与语义空间中的相似度结构趋于一致. 此外, 在哈希码学习 阶段, 引人加权余弦三元组损失进一步利用数据的语义信息;且为减小哈希码的量化损失, 使用离散优化的方法优化 哈希函数. 在 3 个跨模态数据集 MIRFlickr25K, NUS-WIDE, Wikipedia 上, 以不同码位计算 mAP, 且所提方法的 mAP 值均优于其他算法，验证了其在跨模态哈希检索上的优越性、鲁棒性以及 CMPM 的有效性.
\end{abstract}

关键词: 跨模态检索; 映射学习; 对抗学习; 离散哈希

中图法分类号: TP391.41 DOI: 10.3724/SP.J.1089.2021.18599

\section{Adversarial Projection Learning Based Hashing for Cross-Modal Retrieval}

\author{
Zeng Chao ${ }^{1)}$, Bai Cong ${ }^{1)^{*}}$, Ma Qing ${ }^{2)}$, and Chen Shengyong ${ }^{3)}$ \\ 1) (College of Computer Science and Technology, Zhejiang University of Technology, Hangzhou 310023) \\ 2) (College of Science, Zhejiang University of Technology, Hangzhou 310023) \\ 3) (College of Computer Science and Engineering, Tianjin University of Technology, Tianjin 300384)
}

\begin{abstract}
Cross-modal Hashing has received a lot of attentions in the field of cross-modal retrieval due to its high retrieval efficiency and low storage cost. Most of the existing cross-modal Hashing methods learn Hash codes directly from multimodal data and cannot fully utilize the semantic information of the data, so the distribution consistency of low-dimensional features across modalities cannot be guaranteed. To this end, adversarial projection learning based Hashing for cross-modal retrieval (APLH) is proposed, which uses adversarial training to learn low-dimensional features from different modalities and to ensure the distribution consistency of low-dimensional features across modalities. On this basis, cross-modal projection matching constrain (CMPM) is introduced which minimizes the Kullback-Leibler divergence between feature projection matching distributions and label projection matching distributions, and label information is used to align
\end{abstract}

收稿日期：2020-08-24; 修回日期：2020-11-10. 基金项目：国家重点研发计划(2018YFB1305200); 浙江省自然科学基金 (LY18F020032); 国家自然科学基金(U1908210, 61976192). 曾超(1998一), 男, 硕士, CCF 会员, 主要研究方向为多媒体检索、计算机 视觉; 白琮(1981一), 男, 博士, 副教授, 博士生导师, CCF 会员, 论文通讯作者, 主要研究方向为多媒体信息处理、计算机视觉; 马青 (1983一), 女, 硕士, 讲师, 博士生导师, 主要研究方向为多媒体检索、计算机视觉; 陈胜勇(1973一), 男, 博士, 教授, 博士生导师, CCF 会员, 主要研究方向为计算机视觉、图像处理. 
similarities between low-dimensional features of data with similarities in semantic space. Furthermore, in the Hashing learning phase, a weightedcosine triplet loss is introduced to further exploit the semantic information of the data, and to reduce the quantization loss, the Hashing function using a discrete optimization approach is optimized. The mean average precision of the proposed method on three databases MIRFlickr25K, NUS-WIDE and Wikipedia is better than other methods of comparison, which verifies the effectiveness of

CMPM and shows the robustness of our method.

Key words: cross-modal retrieval; projection learning; adversarial learning; discrete Hashing

随着科学技术的发展，人们的日常生活中每 天都会产生大量的多媒体数据, 包括文本、音频、 视频和图像. 同时, 随着多媒体数据的快速增长和 不同模态的数据表示不一致, 对海量异构数据的 高效检索成为一个迫切需求, 也是一个巨大的挑 战. 其中由图像检索文本和由文本检索图像的研 究是最广泛的, 这意味着当用户在以某幅图像或 某段文字进行检索时，期望返回的结果是与查询 目标主题相关的另一模态的数据. 哈希方法是大 规模跨模态检索的普遍解决方案之一, 这类方法 将数据的高维特征转换为二进制编码, 使语义相 似的样本具有相似的编码.

近年来, 人们提出了许多跨模态哈希方法. 其 中比较有代表性的包括语义保留哈希 (semanticspreserving Hashing, SePH) $)^{[1]}$, 集合矩阵分解哈希 (collective matrix factorization Hashing, CMFH) ${ }^{[2]}$, 语义相关最大化(semantic correlation maximization, $\mathrm{SCM})^{[3]}$, 语义主题多模态哈希 (semantic topic multimodal Hashing, STMH) ${ }^{[4]}$. 这些跨模态哈希方法都 使用了手工构建的特征进行哈希函数的学习, 但 是手工特征的提取过程是独立于算法的训练过程, 所以这些方法在实际应用中可能无法展现出令人 满意的性能.

自 AlexNet ${ }^{[5]}$ 于 2012 年提出以来, 深度卷积神 经网络(deep convolutional neural networks, DCNN) 已成功地应用于许多计算机视觉任务 ${ }^{[6-10]}$. 许多研 究也开始探索它在跨模态哈希方法中的作用. 例 如, Zhang 等 ${ }^{[11]}$ 发现应用了 DCNN 的哈希方法能取 得优越的检索性能. 虽然基于 DCNN 的哈希方法 在跨模态检索中取得了显著的成果，但仍有许多 问题需要解决. 首先, 大部分的跨模态哈希方法在 训练过程中没有充分利用标签信息, 导致不同模 态的语义信息没有得到充分的利用. 其次, 由于不 同模态特征的分布不一致, 需要解决异构差异. 另外值得注意的是, 不同模态特征需要具有辨别 性, 即具有相同语义信息的跨模态数据能够在低
维空间内有更近的距离, 反之, 距离更远. 最后, 最优的哈希函数也同样需要保持模态间的语义一 致性.

针对以上问题, 本文提出了一种用于跨模态检 索的基于对抗投影学习的跨模态哈希 (adversarial projection learning based Hashing for cross-modal retrieval, APLH)方法. 该方法由 2 个主要模块组成, 且使用对抗训练的方式学习不同模态的特征表示和 哈希码. 在低维特征学习的模块中采用对抗训练的 方式对 2 种模态进行特征学习, 同时引入跨模态投 影匹配(cross-modal projection matching, CMPM) ${ }^{[12]}$ 最小化特征投影匹配分布和标签投影匹配分布之 间的 KL(Kullback-Leibler)散度, 这样既能充分利 用 2 种模态的语义知识, 又能保持模态间特征表示 的分布一致性. 与特征学习步骤一样, 哈希模块也 采用对抗学习, 进一步保证哈希码在模态间的分 布一致性. 同时, 为了保持哈希码中的语义一致 性, 引人加权余弦三元组损失. 因此, 本文方法不 仅克服了将多模态数据嵌人到共同特征空间时语 义信息丢失的缺陷, 还能拟合不同模态之间的特 征分布, 并且使学习到的哈希码具有优秀的判别 性，还能够不丢失模态间的语义一致性.

\section{1 相关工作}

\section{1 基于无监督学习的跨模态哈希}

基于无监督学习的跨模态哈希方法通过挖掘 不带标签的训练数据在模态内及模态间的信息学 习哈希函数. 例如, STMH 在考虑哈希码隐含性的 同时也保留了其离散性, 使哈希码的每一位都能 够显示出文本或图像是否包含相应的主题. $\mathrm{UGACH}^{[13]}$ 使用了生成对抗网络对跨模态哈希进 行建模, 通过对抗训练学习哈希码并借助关联图 获取具有相似结构的跨模态信息对，从而提高了 检索精度. UDCMH ${ }^{[14]}$ 是第 1 个在无监督深度学习 框架中实现基于矩阵分解的跨模态哈希方法, 其 
使用了一种新型的二值隐式表示模型, 使该方法 能够直接学习离散的哈希码而不需要松驰.

\section{2 基于监督学习的跨模态哈希}

基于监督学习的跨模态哈希方法使用监督信 息(如标签等)提高检索性能. SCM 利用了标签信息 来学习特定模态之间的转换, 从而保证模态之间 的最大相关性. SePH 通过在概率分布中对相似性 矩阵进行建模, 同时最小化 KL 散度生成统一的哈 希码. $\mathrm{DCMH}^{[15]}$ 使用了一个端到端的学习框架，通 过最小化负对数似然损失保持跨模态相似性. $\mathrm{SSAH}^{[16]}$ 是一种新的自监督对抗哈希方法, 也是第 1 个使用对抗性学习解决跨模态哈希问题的方法, 将自我监督的语义学习与对抗学习相结合, 在一 定程度上保持了跨模态数据之间的语义相关性以 及跨模态表达一致性. AGAH ${ }^{[17]}$ 使用一种对抗学习 引导的多标签注意力机制来辅助特征学习的过程, 并且设计了一个非对称哈希网络, 可以保存哈希 码的相似度结构. 总体而言, 无监督的跨模态哈希 方法虽然有着数据易获取、可以减小人为的误差概 率等优点，但是由于没有利用数据的语义信息，其 在检索性能上一般是要差于监督式的跨模态哈希 方法. 与 AGAH方法相比, 本文提出的 APLH 方法 是由 2 步式对抗学习引导的, 并且在特征学习模块 引人了跨模态投影匹配约束，在多标签信息的监 督下可以保证特征的相似性，从而可以进一步最 小化不同模态之间的语义差距.

\section{2 本文方法}

\section{1 问题定义}

与许多跨模态哈希方法一样, 假设有 $n$ 对图像文本对的初始特征. 图像模态的输人表示为 $\boldsymbol{X}=$ $\left[x_{1}, \cdots, x_{n}\right]^{d \times n}$ ，其中， $x_{i}$ 是第 $i$ 幅图像的特征向量; $d$ 为特征向量的维度. 文本模态的输人表示为
$\boldsymbol{Y}=\left[\boldsymbol{y}_{1}, \cdots, \boldsymbol{y}_{n}\right]^{t \times n}$, 与图像模态一样， $\boldsymbol{y}_{i}$ 是第 $i$ 幅图 像对应的文本描述的特征向量： $t$ 是文本特征向量 的维度. 另外, 以一幅图像和其对应的文本描述为 一对, 这 $n$ 对数据的标签表示为 $\boldsymbol{L}=\left[\boldsymbol{I}_{1}, \cdots, \boldsymbol{I}_{n}\right]^{d \times n}$, 其中, $\boldsymbol{I}_{i}=\left[l_{i 1}, \cdots, l_{i c}\right]$ 代表每个标签向量有 $c$ 维, 即 共有 $c$ 类，若该对数据包含其中某一类，则 $\boldsymbol{I}_{i}$ 向量 中对应位置的值为 1 , 否则为 0 . 需要注意的是, 在 本文中定义的标签为多标签向量, 也就是说一幅 图像或一段文本描述可以包含一个或多个标签. 利用给定的训练集 $\boldsymbol{X}, \boldsymbol{Y}$ 以及 $\boldsymbol{L}, \mathrm{APLH}$ 的目标是 学习图像模态和文本模态的 2 个哈希函数 $H_{x}$ 和 $H_{y}$, 以及分别由 2 个哈希函数生成的哈希码 $\boldsymbol{B}_{x}=$ $\left[\boldsymbol{b}_{1}^{x}, \cdots, \boldsymbol{b}_{n}^{x}\right]^{k \times n}$ 和 $\boldsymbol{B}_{y}=\left[\boldsymbol{b}_{1}^{y}, \cdots, \boldsymbol{b}_{n}^{y}\right]^{k \times n}$, 其中, $\boldsymbol{b}_{i}^{x} \in\{-1$, $+1\}^{k}$ 与 $\boldsymbol{b}_{i}^{y} \in\{-1,+1\}^{k}$ 都是 $k$ 维的离散向量.

\section{2 框架概述}

本文方法如图 1 所示，由 2 个部分构成.

(1) 特征学习模块. 对于图像模态, 通过卷积 神经网络将图像处理为 4096 维的特征向量, 再经 过多层全连接层将特征向量嵌人到低维特征空间. 对于文本模态, 文本特征网络中的多层全连接层 将原始的文本特征嵌人到低维特征空间. 在这个 模块中, 通过引人对抗训练和 CMPM 约束, 弥合 文本模态和图像模态在低维特征空间的概率分布 之间的差异，同时保存语义信息.

(2) 哈希码学习模块. 输人即特征学习模块输 出的低维特征向量, 在这一步分别经过一层全连 接层后通过 $\tanh$ 激活函数输出伪哈希码, 在检索 阶段再将伪哈希码通过符号函数处理得到哈希码. 引人了对抗训练, 并引人加权三元组损失保持模 态间的语义一致性. 同时引人简单的线性分类器 保存语义信息, 在训练过程中不断更新分类器的 参数及哈希码的最优解, 使哈希函数在减小量化 损失的情况下还能生成具有辨别性的哈希码.

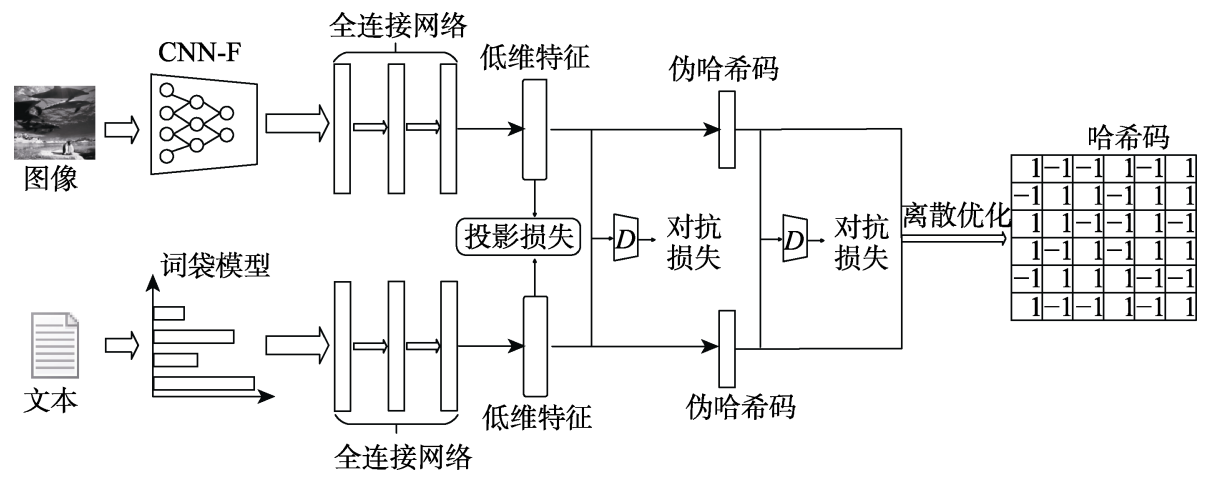

图 1 本文方法的框架 


\section{3 特征学习}

定义 $\boldsymbol{f}^{x}=F_{x}\left(\boldsymbol{X} ; \boldsymbol{\theta}_{F_{x}}\right)$ 和 $\boldsymbol{f}^{y}=F_{y}\left(\boldsymbol{Y} ; \boldsymbol{\theta}_{F_{y}}\right)$ 为图像 模态和文本模态在低维特征空间的投影, $F_{x}$ 和 $F_{y}$ 分别代表了图像特征网络与文本特征网络, $\boldsymbol{\theta}_{F_{\mathrm{X}}}$ 和 $\theta_{F_{y}}$ 代表了它们的参数. 图像特征网络由 DCNN 和 多层全连接网络构成, 文本特征网络由 3 个全连接 层构成. 对于图像特征网络, 由于在训练过程中会 包含大量的穴余信息, 因此本文方法使用迁移学 习, 将在 ImageNet 上预训练的 $\mathrm{CNN}-\mathrm{F}^{[18]}$ 的参数固 定, 在训练过程中仅更新最后几层全连接层, 这样 在减少冗余信息的同时也能大大提升训练的速度.

\subsection{1 对抗训练}

为了弥合模态间的低维特征分布的差异，本 文方法设计了一个判别器 $D_{\mathrm{F}}$, 利用判别器 $D_{\mathrm{F}}$ 对 低维特征向量进行判别: 如果该低维特征来自图 像模态, 则判定为真; 若来自文本模态, 则判定为 假. 当判别器 $D_{\mathrm{F}}$ 试图区分未知模态的低维特征时, 特征网络 $F_{x}$ 和 $F_{y}$ 试图混淆判别器 $D_{\mathrm{F}}$. 上述过程 可以定义交叉熵损失

$$
\begin{gathered}
\mathcal{L}_{\mathrm{F}}=-\frac{1}{n} \sum_{i=1}^{n}\left(\ln \left(D_{\mathrm{F}}\left(\boldsymbol{f}_{i}^{x} ; \boldsymbol{\theta}_{D_{\mathrm{F}}}\right)\right)+\right. \\
\ln \left(1-D_{\mathrm{F}}\left(\boldsymbol{f}_{i}^{y} ; \boldsymbol{\theta}_{D_{\mathrm{F}}}\right)\right)
\end{gathered}
$$

其中, $D_{\mathrm{F}}\left(\boldsymbol{f}_{i}^{x} ; \boldsymbol{\theta}_{D_{\mathrm{F}}}\right)$ 和 $D_{\mathrm{F}}\left(\boldsymbol{f}_{i}^{y} ; \boldsymbol{\theta}_{D_{\mathrm{F}}}\right)$ 都是判别器对输 人给出的其来自图像模态的概率, 其范围为 $(0,1), \theta_{D_{\mathrm{F}}}$ 是判别器 $D_{\mathrm{F}}$ 的参数. 判别器给出的概 率值越高, 判别器对输入的特征模态判断越倾向 于图像模态，反之就越倾向于文本模态.

\subsection{2 跨模态投影匹配}

为了保证在嵌人到低维特征空间时依然不丢 失数据的语义信息, 同时进一步弥合模态间的低 维特征分布的差异, 本文方法引人了一种图像-文 本匹配约束, 即 CMPM 约束, 该约束定义了特征 投影匹配分布和标签投影匹配分布之间的 KL 散 度，用于衡量 2 个分布的相似度，以此来关联不同 模态嵌人低维特征空间的表示. 当给定一个包含 $n$ 对图像-文本对的低维特征的小批次时, 定义一个 三元组构造 $\left\{\boldsymbol{f}_{i}^{x}, \boldsymbol{f}_{j}^{y}, o_{i, j}\right\}$, 其中, $o_{i, j}$ 为第 $i$ 幅图像 和第 $j$ 个文本的标签向量 $\boldsymbol{I}_{i}$ 和 $\boldsymbol{l}_{j}$ 之间的内积, 可以 理解为它们之间的相似度, 其定义为

$$
o_{i, j}=\boldsymbol{I}_{i}^{\mathrm{T}} \cdot \boldsymbol{I}_{j}
$$

显而易见，当来自 2 个模态的输人之间没有一
个标签是相同的时候, $o_{i, j}=0$; 反之 $o_{i, j}$ 的值在 $\left[1, \min \left(\left\|\boldsymbol{I}_{i}\right\|, \| \boldsymbol{I}_{j}||\right)\right]$ 范围内. 而一般的跨模态哈希对多 标签数据之间的相似度定义为

$$
o_{i, j}=\left\{\begin{array}{l}
1,\left\|\boldsymbol{l}_{i}^{\mathrm{T}} \cdot \boldsymbol{l}_{j}\right\| \neq 0 \\
0,\left\|\boldsymbol{l}_{i}^{\mathrm{T}} \cdot \boldsymbol{l}_{j}\right\|=0
\end{array}\right.
$$

而这样的定义对于多标签数据集来说是不合 适的. 于是，对于多标签投影匹配分布，定义为一 个归一化的概率输出, 表示为

$$
p_{i, j}=\frac{o_{i, j}}{\sum_{k=1}^{n} o_{i, k}}
$$

标签投影匹配分布对于特征投影匹配分布来 说，是属于数据在语义空间上真实的概率分布，并 且不会随网络更新而改变. 而特征投影匹配分布 则是本文方法需要学习的对象, 其定义为 Softmax 函数的输出, 即

$$
q_{i, j}=\frac{\exp \left(\left(\boldsymbol{f}_{i}^{x}\right)^{\mathrm{T}} \overline{\boldsymbol{f}}_{j}^{y}\right)}{\sum_{k=1}^{n} \exp \left(\left(\boldsymbol{f}_{i}^{x}\right)^{\mathrm{T}} \overline{\boldsymbol{f}}_{k}^{y}\right)}
$$

其中, $\overline{\boldsymbol{f}}_{j}^{y}=\boldsymbol{f}_{j}^{y} /\left\|\boldsymbol{f}_{j}^{y}\right\|$ 是归一化的文本特征向量. 这里 $\left(\boldsymbol{f}_{i}^{x}\right)^{\mathrm{T}} \overline{\boldsymbol{f}}_{j}^{y}$ 代表第 $i$ 幅图像的特征在归一化的第 $j$ 个文本特征上的投影标量; $q_{i, j}$ 可以看做在一个 小批次中所有来自特征对集合 $\left\{\boldsymbol{f}_{i}^{x}, \boldsymbol{f}_{j}^{y}\right\}_{j=1}^{n}$ 中的投 影标量的概率分布. 显然, 2 个特征向量越相似, 其投影标量就越大, 反之越小. 结合 $p_{i, j}$ 和 $q_{i, j}$ 这 2 个分布, 求 $p_{i, j}$ 到 $q_{i, j}$ 的 KL 散度, 通俗来讲, 就是 求图像特征 $\boldsymbol{f}_{i}{ }^{x}$ 与正确匹配的文本特征之间的关联 损失，定义为

$$
\mathcal{L}_{\mathrm{p}_{\mathrm{izt}}}=\frac{1}{n} \sum_{i=1}^{n} \sum_{j=1}^{n} q_{i, j} \ln \left(\frac{q_{i, j}}{p_{i, j}+\delta}\right)
$$

其中, $\delta$ 是极小的微扰项, 用于防止出现 $p_{i, j}=0$ 导致训练发生错误的情况; $\mathcal{L}_{\mathrm{P}_{\mathrm{i} 2 \mathrm{t}}}$ 是由图像特征投 影到文本特征时的损失. 同理，由文本特征投影到 图像特征时损失为 $\mathcal{L}_{\mathrm{p}_{\mathrm{rzi}}}$, 其定义与 $\mathcal{L}_{\mathrm{p}_{\mathrm{izt}}}$ 类似. 所以 总的跨模态投影损失定义为

\section{4 哈希学习}

$$
\mathcal{L}_{\mathrm{p}}=\mathcal{L}_{\mathrm{p}_{\mathrm{i} 2 \mathrm{t}}}+\mathcal{L}_{\mathrm{p}_{\mathrm{t} 2 \mathrm{i}}}
$$

在此模块中，本文方法引入了多个损失函数 以获得最优的哈希函数. 2 个哈希函数 $H_{x}$ 和 $H_{y}$ 分 别属于图像模态和文本模态, 均由一层全连接网 
络组成. 原始输人经过图像特征网络和文本特征 网络后被嵌人到低维特征空间, 得到 $\boldsymbol{f}^{x}$ 和 $\boldsymbol{f}^{y}$. 低 维特征作为哈希函数的输人, 得到潜在空间的伪 哈希码 $\widetilde{\boldsymbol{B}}^{x}=H_{x}\left(\boldsymbol{f}^{x} ; \boldsymbol{\theta}_{H_{x}}\right)$ 和 $\widetilde{\boldsymbol{B}}^{y}=H_{y}\left(\boldsymbol{f}^{y} ; \boldsymbol{\theta}_{H_{y}}\right)$, 此 时的伪哈希码是由 $\tanh$ 函数激活后的向量. 当进 行检索时, 伪哈希码经由符号函数处理得到离散 的哈希码 $\boldsymbol{B}^{x}$ 和 $\boldsymbol{B}^{y}$. 符号函数的定义为

$$
\operatorname{sign}(x)= \begin{cases}+1, & x \geqslant 0 \\ -1, & x<0\end{cases}
$$

本文方法在哈希学习引人了对抗训练, 目标 是达到与特征学习中的对抗训练同样的效果, 即 弥合不同模态伪哈希码分布的差异.类似于特征学 习中的设计, 假设判别器为 $D_{\mathrm{H}}$. 这里的对抗损失 为 $\mathcal{L}_{\mathrm{H}}$, 与 $\mathcal{L}_{\mathrm{F}}$ 相似, 定义为

$$
\begin{gathered}
\mathcal{L}_{\mathrm{H}}=-\frac{1}{n} \sum_{i=1}^{n}\left(\ln \left(D_{\mathrm{H}}\left(\tilde{\boldsymbol{b}}_{i}^{x} ; \boldsymbol{\theta}_{D_{\mathrm{H}}}\right)\right)+\right. \\
\ln \left(1-D_{\mathrm{H}}\left(\tilde{\boldsymbol{b}}_{i}^{y} ; \boldsymbol{\theta}_{D_{\mathrm{H}}}\right)\right)
\end{gathered}
$$

其中, $\theta_{D_{\mathrm{H}}}$ 是判别器 $D_{\mathrm{H}}$ 的参数. 当判别器 $D_{\mathrm{H}}$ 判定 $\tilde{\boldsymbol{b}}_{i}^{x}$ 为真, $\tilde{\boldsymbol{b}}_{i}^{y}$ 为假时, 哈希函数 $H_{x}$ 和 $H_{y}$ 试图混淆 判别器 $D_{\mathrm{H}}$.

\subsection{1 加权余弦三元组损失}

为了在哈希码中保持模态间的相似度结构, 本文方法引人了加权三元组损失函数. 每个三元 组都由一个针点(查询), 以及正样本和负样本(待 查询)组成. 当由图像检索文本时, 三元组一般定 义为 $\left\{\tilde{\boldsymbol{b}}_{i}^{X}, \tilde{\boldsymbol{b}}_{j}^{y+}, \tilde{\boldsymbol{b}}_{k}^{y-}\right\}$; 当由文本检索图像时, 三元 组定义为 $\left\{\tilde{\boldsymbol{b}}_{i}^{y}, \tilde{\boldsymbol{b}}_{j}^{x+}, \tilde{\boldsymbol{b}}_{k}^{x-}\right\}$. 若样本与针点拥有至少 一个相同的标签, 则为正样本, 反之为负样本. 同 时, 考虑多标签情况下, 在语义空间的相似度是具 有排序性的, 不像单标签的相似度仅被定义为 $\{0,1\}$, 于是本文方法引人了权重因子保证多标签 数据在语义空间的相似度结构. 加权余弦三元组 损失, 就是将跨模态数据之间的相似度用余弦距离 来度量(即越相似余弦距离越小, 反之越大), 并且 对每一个三元组乘上一个权重因子, 其定义为

$$
\begin{aligned}
& \mathcal{L}_{\text {triplet }}^{\mathrm{i2t}}=\sum_{i, j, k} \omega\left(r_{j}, r_{k}\right)\left(\operatorname { m a x } \left(\cos \left(\tilde{\boldsymbol{b}}_{i}^{X}, \tilde{\boldsymbol{b}}_{k}^{y-}\right)-\right.\right. \\
& \left.\left.\cos \left(\tilde{\boldsymbol{b}}_{i}^{X}, \tilde{\boldsymbol{b}}_{j}^{y+}\right)+m, 0\right)\right) \\
& \mathcal{L}_{\text {triplet }}^{\mathrm{t2i}}=\sum_{i, j, k} \omega\left(r_{j}, r_{k}\right)\left(\operatorname { m a x } \left(\cos \left(\tilde{\boldsymbol{b}}_{i}^{y}, \tilde{\boldsymbol{b}}_{k}^{X-}\right)-\right.\right. \\
& \left.\left.\cos \left(\tilde{\boldsymbol{b}}_{i}^{y}, \tilde{\boldsymbol{b}}_{j}^{X+}\right)+m, 0\right)\right)
\end{aligned}
$$

式(10)代表查询目标是图像时的加权余弦三元组 损失函数; 式(11)代表查询目标是文本时的加权余 弦三元组损失函数. 其中, 定义为

$$
\omega\left(r_{j}, r_{k}\right)=\frac{2^{r_{j}}-2^{r_{k}}}{Z}
$$

这里 $r_{j}$ 代表了相似样本与针点相同标签个数与针 点标签个数之比, 在通常情况下 $r_{k}=0, Z$ 为使权 重因子归一化的常数, 计算式为

$$
Z @ p=\sum_{i=1}^{p} \frac{2^{r_{i}}-1}{\ln (1+i)}
$$

其中, $r_{i}$ 是在待查询集正确排序后得到的, 所以总 的三元组损失为

$$
\mathcal{L}_{\text {triplet }}=\mathcal{L}_{\text {triplet }}^{\mathrm{i} 2 \mathrm{t}}+\mathcal{L}_{\text {triplet }}^{\mathrm{t} \mathrm{i}}
$$

\subsection{2 基于线性分类器的离散哈希}

为了在哈希码中保留标签的语义信息, 本文 方法将 $\mathrm{FSDH}^{[19]}$ 扩展到跨模态哈希, 并同样使用标 签信息对原始数据在汉明空间的表示进行分类. 目标函数定义为

$$
\begin{aligned}
& \min _{\boldsymbol{B}^{x}, H_{x}, \boldsymbol{W}}\left\|\boldsymbol{B}^{\boldsymbol{x}}-\boldsymbol{L} \boldsymbol{W}\right\|_{\mathrm{F}}^{2}+\lambda\|\boldsymbol{W}\|_{\mathrm{F}}^{2} ; \\
& \min _{\boldsymbol{B}^{y}, H_{y}, \boldsymbol{W}}\left\|\boldsymbol{B}^{y}-\boldsymbol{L} \boldsymbol{W}\right\|_{\mathrm{F}}^{2}+\lambda\|\boldsymbol{W}\|_{\mathrm{F}}^{2} .
\end{aligned}
$$

其中， $\boldsymbol{B}^{x}=\operatorname{sign}\left(\widetilde{\boldsymbol{B}}^{x}\right) ; \quad \boldsymbol{B}^{y}=\operatorname{sign}\left(\widetilde{\boldsymbol{B}}^{y}\right) ; \boldsymbol{W} \in \mathbb{R}^{C \times K}$ 是线性投影矩阵; $\boldsymbol{L}$ 是标签矩阵; $\lambda$ 是正则化参 数. 由于 $\boldsymbol{B}^{x}$ 和 $\boldsymbol{B}^{y}$ 是哈希函数 $H_{x}$ 和 $H_{y}$ 的输出经过 符号函数得来, 元素仅为 1 和 -1 , 使上述函数的优 化成为一个 NP-hard 问题. 很多方法在训练过程中 直接令 $\boldsymbol{B}^{x}=\widetilde{\boldsymbol{B}}^{x}, \boldsymbol{B}^{y}=\widetilde{\boldsymbol{B}}^{y}$, 这样虽然简化了计算 量, 但此松他破坏了哈希码的离散性, 往往造成了 大量的量化损失. 所以，为了解决这个 NP-hard 问 题, 也为了在模态间学习统一的哈希码, 本文方法 引人 $\boldsymbol{B}=\boldsymbol{B}^{x}=\boldsymbol{B}^{y}$. 并且为了减小量化损失, 在目 标函数中分别加人 $\left\|\boldsymbol{B}-\widetilde{\boldsymbol{B}}^{x}\right\|_{F}^{2}$ 和 $\left\|_{\boldsymbol{B}}-\widetilde{\boldsymbol{B}}^{y}\right\|_{\mathrm{F}}^{2}$, 最小化 这 2 项的最终作用就是使 $\boldsymbol{B}=\widetilde{\boldsymbol{B}}^{x}=\widetilde{\boldsymbol{B}}^{y}$. 更改后的 目标函数为

$$
\begin{array}{r}
\min _{\boldsymbol{B}, H_{x}, \boldsymbol{W}}\|\boldsymbol{B}-\boldsymbol{L} \boldsymbol{W}\|_{\mathrm{F}}^{2}+\lambda\|\boldsymbol{W}\|_{\mathrm{F}}^{2}+\mu\left\|\boldsymbol{B}-\widetilde{\boldsymbol{B}}^{x}\right\|_{\mathrm{F}}^{2} ; \\
\min _{\boldsymbol{B}, H_{y}, \boldsymbol{W}}\|\boldsymbol{B}-\boldsymbol{L} \boldsymbol{W}\|_{\mathrm{F}}^{2}+\lambda\|\boldsymbol{W}\|_{\mathrm{F}}^{2}+v\left\|\boldsymbol{B}-\widetilde{\boldsymbol{B}}^{y}\right\|_{\mathrm{F}}^{2} .
\end{array}
$$

其中, $\boldsymbol{B} \in\{-1,1\}^{N \times K} ; \mu$ 为一个 $(0,1)$ 的常数; $v=1-\mu$. 将上述函数合并, 即离散优化的总损失 
函数为

$$
\mathcal{L}_{\mathrm{q}}=\|\boldsymbol{B}-\boldsymbol{L} \boldsymbol{W}\|_{\mathrm{F}}^{2}+\lambda\|\boldsymbol{W}\|_{\mathrm{F}}^{2}+\mu\left\|\boldsymbol{B}-\widetilde{\boldsymbol{B}}^{x}\right\|_{\mathrm{F}}^{2}+v\left\|\boldsymbol{B}-\widetilde{\boldsymbol{B}}^{y}\right\|_{\mathrm{F}}^{2}
$$

\section{5 算法优化}

总的损失函数为

$$
\begin{gathered}
\mathcal{L}_{\text {adv }}=\mathcal{L}_{\mathrm{F}}+\mathcal{L}_{\mathrm{H}} \\
\mathcal{L}_{\text {total }}=\alpha \mathcal{L}_{\mathrm{adv}}+\eta \mathcal{L}_{\mathrm{p}}+\gamma \mathcal{L}_{\text {triplet }}+\beta \mathcal{L}_{\mathrm{q}}
\end{gathered}
$$

其中， $\mathcal{L}_{\mathrm{adv}}$ 表示总的对抗损失. 由于在损失函数 $\mathcal{L}_{\mathrm{q}}$ 中有 2 个独立的矩阵变量 $\boldsymbol{B}$ 和 $\boldsymbol{W}$ ，因此整体的 优化问题就变为非凸问题，直接优化较为困难，所 以本文方法采用交替方向法将优化问题分为 2 步. 具体的流程如下.

\subsection{1 深度网络参数优化}

固定矩阵变量 $\boldsymbol{B}$ 和 $\boldsymbol{W}$ ，使用随机梯度下降优 化算法优化深度网络的参数. 在使用随机梯度下 降算法对总的损失函数 $\mathcal{L}_{\text {total }}$ 求梯度后, 再利用反 向传播更新深度网络的参数 $\theta_{D_{\mathrm{F}}}, \theta_{D_{\mathrm{H}}}, \theta_{F_{x}}, \theta_{F_{y}}$, $\boldsymbol{\theta}_{H_{x}}, \boldsymbol{\theta}_{H_{y}}$. 由于随机梯度下降算法在深度学习中 被广泛应用，这里不再赘述.

\subsection{2 矩阵变量更新}

当所有数据训练后，再通过固定深度网络参 数以及任意其中一个矩阵变量, 更新另一个矩阵 变量, 直到 2 个矩阵变量都更新, 开始下一次训练, 直到总的损失函数收敛.

(1) 固定哈希码矩阵变量 $\boldsymbol{B}$. 将式(15)对 $\boldsymbol{W}$ 求 偏导并使之等于 0 , 即

$$
\frac{\partial \mathcal{L}_{\mathrm{q}}}{\partial \boldsymbol{W}}=-\boldsymbol{B}^{\mathrm{T}} \boldsymbol{B}-2 \boldsymbol{L}^{\mathrm{T}} \boldsymbol{B}+2 \boldsymbol{L}^{\mathrm{T}} \boldsymbol{L} \boldsymbol{W}+2 \lambda \boldsymbol{W}=0
$$

可以得到 $W$ 的闭式解为

$$
\boldsymbol{W}=\left(\boldsymbol{L}^{\mathrm{T}} \boldsymbol{L}+\lambda \boldsymbol{I}\right)^{-1} \boldsymbol{L}^{\mathrm{T}} \boldsymbol{B}
$$

(2) 固定矩阵变量 $\boldsymbol{W}$. 将对式(15)进行整理 后, 由于 $\boldsymbol{B} \in\{-1,1\}^{N \times K}$, 使 $-\operatorname{tr}\left(\boldsymbol{B}^{\mathrm{T}} \boldsymbol{B}\right)$ 的项为常数, 所以式(15)简化为

$$
\min _{\boldsymbol{B}}-\operatorname{tr}\left(\boldsymbol{B}^{\mathrm{T}}\left(\boldsymbol{L} \boldsymbol{W}+\mu \widetilde{\boldsymbol{B}}^{x}+v \widetilde{\boldsymbol{B}}^{y}\right)\right)
$$

可以得到 $\boldsymbol{B}$ 的离散解为

\section{6 算法实现步骤}

$$
\boldsymbol{B}=\operatorname{sign}\left(\boldsymbol{L} \boldsymbol{W}+\mu \widetilde{\boldsymbol{B}}^{x}+v \widetilde{\boldsymbol{B}}^{y}\right)
$$

本文算法实现步骤如下.

输人. 训练集 $\left\{\boldsymbol{x}_{i}, \boldsymbol{y}_{i}, \boldsymbol{l}_{i}\right\}_{i=1}^{n}$, batch size $m$, 哈希 码长 $K$.

输出. 最优的网络参数 $\boldsymbol{\theta}_{F_{x}}, \boldsymbol{\theta}_{F_{y}}, \boldsymbol{\theta}_{H_{x}}, \boldsymbol{\theta}_{H_{y}}$ 和哈 希码 $\boldsymbol{B}$

Step1. 初始化深度网络参数, 用标准正态分布初
始化哈希码矩阵 $\boldsymbol{B}$.

Step2. Repeat

Step2.1. for $i=1$ to $\left\lceil\frac{n}{m}\right\rceil$ do

用第 2.5.1 节中的方式更新深度网络的参数;

Step2.2. 用式(19)对 $\boldsymbol{W}$ 进行更新;

Step2.3. 用式(21)对 $\boldsymbol{B}$ 进行更新.

Step3. 直到总损失函数的收玫阈值或迭代次数上限.

\section{3 实验与分析}

本文在 MIRFlickr25K ${ }^{[20]}$, NUS-WIDE ${ }^{[21]}$ 和 Wikipedia $^{[22]} 3$ 个常用的跨模态数据集上进行了实 验, 与最近 6 种先进的跨模态哈希方法 $(\mathrm{SePH}$, $\mathrm{PRDH}^{[23]}$, DCMH, SSAH, AGAH, ECMH ${ }^{[24]}$ )在 2 个 跨模态检索任务(以图像检索文本和以文本检索图 像)上进行了比较, 并且设计了消融实验以证明引 人跨模态投影匹配约束的有效性.

\section{1 数据集及评价指标}

MIRFlickr25K 是一个由 25000 幅图像组成的 数据集, 每幅图像都有对应的多个文本标记. 另外 每幅图像有 24 个标签中的一个或多个标签. 在本 文实验中，与 $\mathrm{AGAH}$ 及其他对比方法中的实验设 置相同, 仅选择至少有 20 个文本标记的图像-文本 对作为实验数据. 每幅图像的文本都表示为一个 1386 维词袋(bag-of-words, BOW)模型向量. 本文 随机选择 2000 对作为测试集, 剩下的作为待查询 集, 并且从待查询集中随机抽取 10000 对作为训 练集.

NUS-WIDE 数据集包含 269648 个带有文本标 记的网络图像. 其中每幅图有 81 个标签中的一个 或多个标签. 在本文中, 与 $\mathrm{AGAH}$ 及其他对比方 法中的设置相同, 本文选择了 195834 个属于 21 个最常见标签的图像-文本对, 每幅图像的文本被 表示为一个 1000 维 BOW 向量. 本文实验从该数 据集中随机抽取 2100 对作为测试集, 其余的作为 待查询集, 并以待查询集中随机抽取的 10000 对 作为训练集.

Wikipedia 是一个由 2866 个图像-文本对组成 的跨模态数据集. 每对图像-文本对拥有 10 个标签 中的某一个标签. 每幅图像的文本都被表示为一 个 1000 维 BOW 向量. 本文随机选择 693 对作为 测试集, 剩余 2173 对作为待查询集, 由于该数据 集很小, 所以待查询集也作训练集.

本文利用平均准确率均值(mean average precision, mAP)作为实验的评价指标. 当给定一个查 
询时, 根据汉明距离对待查询集进行排序. 定义在 待查询集第 $k$ 个位置的精度为

$$
P(k)=\frac{\sum_{i=1}^{k} r(i)}{k}
$$

当排序结果中 $i$ 个数据与查询至少拥有一个相 同标签时, $r(i)=1$, 否则 $r(i)=0 . \mathrm{mAP}$ 的计算式为

$$
\mathrm{mAP}=\frac{1}{Q} \sum_{i=1}^{Q} \frac{1}{m_{i}} \sum_{j=1}^{n} P_{i}(j) r_{i}(j)
$$

其中, $Q$ 为查询集的大小; $m_{i}$ 为待查询集中与查 询集中的第 $i$ 项相关的项数; $n$ 为待查询集的大小.

\section{2 实验结果与分析}

将本文方法与最近 6 种先进的跨模态哈希方 法进行比较. 为了公平, 图像模态的输人均使用 CNN-F 提取的 4096 维图像特征. 经过对超参数的 实验分析, 本文设置 $\alpha=\lambda=\beta=1, \eta=\gamma=10, \mu=$ 0.5 . 另外, batch size 为 128 , 总 epoch 为 300 . 对于 MIRFlickr25K 数据集和 Wikipedia 数据集, 初始学 习率为 0.0001 , NUS-WIDE 为 0.00001 .

\subsection{1 消融实验}

本文以哈希码长度为 64 时在 3 个数据集上做 了消融实验，消融实验的结果如表 1 所示. 可以看 出, 引入跨模态投影匹配约束后在不同的数据集 上检索性能有不同幅度的提升. 在图像检索文本 的任务中, 与不加人跨模态投影匹配约束对比, 在 MIRFlickr25K, NUS-WIDE, Wikipedia 数据集上的 $\mathrm{mAP}$ 分别有 $2.5 \%, 0.3 \%, 2.0 \%$ 的提升. 而在文本检 索图像的任务中，在 MIRFlickr25K, NUS-WIDE, Wikipedia 数据集上的 mAP 分别有 $1.6 \%, 5.0 \%$, $1.0 \%$ 的提升. 不难发现, 加人了跨模态投影匹配
约束以后, 本文方法在 3 个数据集上的提升明显, 主要原因是 CMPM 约束关联了具有高相关性的跨 模态数据, 使跨模态的语义信息在低维特征空间 得到有效的保存, 同时在对抗训练的基础上，进一 步弥合了异构数据的低维特征分布差异.

表 1 消融实验的 mAP 对比

\begin{tabular}{clccc}
\hline 任务 & \multicolumn{1}{c}{ 方法 } & MIRFlickr25K & NUS-WIDE & Wikipedia \\
\hline \multirow{2}{*}{ 图检文 } & Base & 0.8211 & 0.6612 & 0.5516 \\
& Base+CMPM & 0.8418 & 0.6720 & 0.5738 \\
\hline \multirow{2}{*}{ 文检图 } & Base & 0.8101 & 0.6828 & 0.8615 \\
& Base+CMPM & 0.8231 & 0.7198 & 0.8785 \\
\hline
\end{tabular}

\subsection{2 对比实验}

本文以哈希码长度为 $16,32,64$ 位时在 3 个数 据集上与其他方法做了对比实验，实验结果如表 2 所示. 可以看出, 本文方法在 3 个数据集上都实现 了最优的检索性能. 对于图像检索文本任务, 在 MIRFlickr25K, NUS-WIDE, Wikipedia 数据集上, 相对于次优的方法, 本文方法在哈希码长度为 16 时 $\mathrm{mAP}$ 分别有了 $2.0 \%, 0.5 \%, 7.0 \%$ 的提升; 在哈希 码长度为 32 时 $\mathrm{mAP}$ 分别有 $4.7 \%, 0.5 \%, 10.5 \%$ 的提 升; 在哈希码长度为 64 时 $\mathrm{mAP}$ 分别有 $4.3 \%, 3.1 \%$, $5.9 \%$ 的提升. 对于文本检索图像的任务, 在哈希 码长度为 16 时 $\mathrm{mAP}$ 分别有 $1.7 \%, 5.5 \%, 10.7 \%$ 的提 升; 在哈希码长度为 32 时 $\mathrm{mAP}$ 分别有 $3.0 \%, 6.6 \%$, $9.2 \%$ 的提升; 在哈希码长度为 64 时 $\mathrm{mAP}$ 分别有 $2.3 \%, 13.6 \%, 9 \%$ 的提升.

\subsection{3 超参数分析}

对于大部分使用深度学习的方法, 超参数是 一个影响模型性能的重要因素, 所以为了实现最 佳的性能, 选择合适的超参数是必要的. 本文针对

\begin{tabular}{|c|c|c|c|c|c|c|c|c|c|c|}
\hline \multirow{2}{*}{ 任务 } & \multirow{2}{*}{ 方法 } & \multicolumn{3}{|c|}{ MIRFlickr25K } & \multicolumn{3}{|c|}{ NUS-WIDE } & \multicolumn{3}{|c|}{ Wikipedia } \\
\hline & & 16 位 & 32 位 & 64 位 & 16 位 & 32 位 & 64 位 & 16 位 & 32 位 & 64 位 \\
\hline \multirow{7}{*}{ 图检文 } & $\mathrm{SePH}^{[1]}$ & 0.6707 & 0.6736 & 0.6747 & 0.5065 & 0.5140 & 0.5189 & 0.5180 & 0.5120 & 0.5460 \\
\hline & $\mathrm{PRDH}^{[23]}$ & 0.6952 & 0.7072 & 0.7108 & 0.5919 & 0.6059 & 0.6116 & & & \\
\hline & $\mathrm{DCMH}^{[15]}$ & 0.7201 & 0.7287 & 0.7418 & 0.5657 & 0.6007 & 0.6001 & 0.4750 & 0.5040 & 0.5050 \\
\hline & SSAH $^{[16]}$ & 0.7709 & 0.7840 & 0.7924 & 0.6020 & 0.6219 & 0.6463 & & & \\
\hline & $\mathrm{AGAH}^{[17]}$ & 0.7923 & 0.7945 & 0.8069 & 0.6455 & 0.6660 & 0.6512 & 0.5104 & 0.5145 & 0.5402 \\
\hline & $\mathrm{ECMH}^{[24]}$ & 0.7569 & 0.7729 & 0.7959 & & & & & & \\
\hline & 本文 & 0.8104 & 0.8322 & 0.8418 & 0.6493 & 0.6692 & 0.6720 & 0.5490 & 0.5687 & 0.5738 \\
\hline \multirow{7}{*}{ 文检图 } & $\mathrm{SePH}^{[1]}$ & 0.6907 & 0.6961 & 0.6990 & 0.5334 & 0.5437 & 0.5499 & 0.7540 & 0.7800 & 0.7860 \\
\hline & $\mathrm{PRDH}^{[23]}$ & 0.7626 & 0.7718 & 0.7755 & 0.6155 & 0.6286 & 0.6349 & & & \\
\hline & $\mathrm{DCMH}^{[15]}$ & 0.7533 & 0.7593 & 0.7730 & 0.5336 & 0.5871 & 0.5916 & 0.8230 & 0.8230 & 0.7890 \\
\hline & $\mathrm{SSAH}^{[16]}$ & 0.7681 & 0.7737 & 0.7838 & 0.6123 & 0.6369 & 0.6398 & & & \\
\hline & $\mathrm{AGAH}^{[17]}$ & 0.7887 & 0.7904 & 0.8049 & 0.6313 & 0.6422 & 0.6336 & 0.7805 & 0.8007 & 0.8058 \\
\hline & $\mathrm{ECMH}^{[24]}$ & 0.7787 & 0.7939 & 0.7985 & & & & & & \\
\hline & 本文 & 0.8019 & 0.8140 & 0.8231 & 0.6660 & 0.6847 & 0.7198 & 0.8635 & 0.8742 & 0.8785 \\
\hline
\end{tabular}

表 2 本文方法与其他 6 种方法的 mAP 对比 
$\gamma, \eta, \mu$ 这 3 个超参数, 在 MIRFlickr25K 数据集 上对长度为 64 位的哈希码做了分析实验, 其中, $\gamma$ 和 $\eta$ 分别控制加权余弦三元组损失和跨模态投影 匹配损失在总损失函数中的比重; $\mu$ 控制图像伪 哈希码和文本伪哈希码对最优哈希码的贡献比重. 本文分别设置 $\mu \in\{0,0.2,0.5,0.8,1.0\}$ 以及 $\gamma, \eta \in\{0,1.0,10.0,100.0,1000.0\}$ 进行实验. 其他 参数在本次实验中均使用第 3.2 节中的设置. 图 2 图 4 显示了各参数在 MIRFlickr25K 数据集上的结 果. 可以发现，当 $\mu=0.5$ 时，本文方法取得了最优 的综合表现，但无论 $\mu$ 值逐渐趋近于 1 还是 0 时, 2 个检索任务的性能增加或衰减的范围都很小. 对 于 $\gamma$ 和 $\eta$ 这 2 个参数, 最优的综合表现出现在 $\eta=\gamma=10$, 这时这 2 个损失函数在总损失函数中 有相对合理的比重. 当这 2 个参数的取值在 $[10$, 1000]范围内，检索性能实际上波动不大.

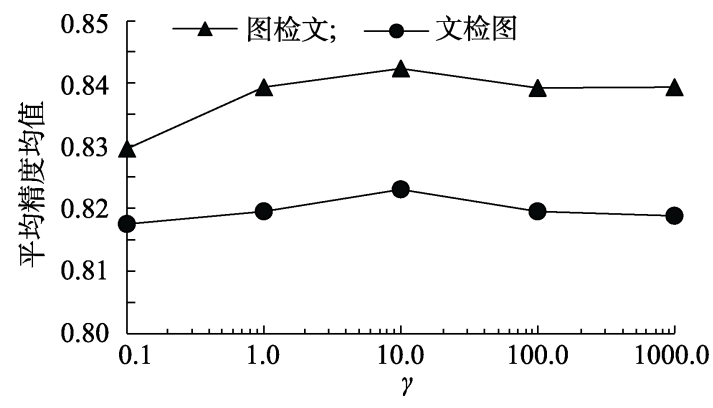

图 264 位码下超参数 $\gamma$ 不同时的 mAP

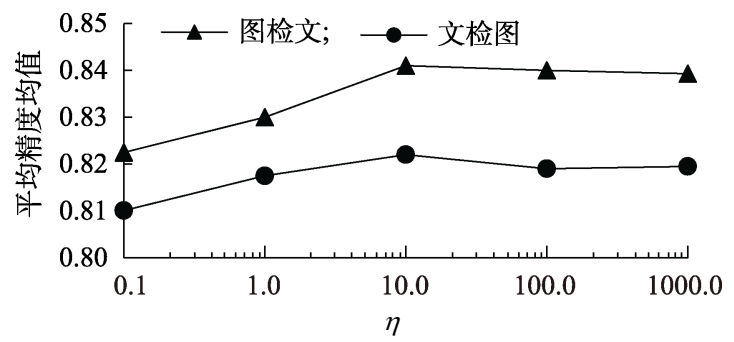

图 364 位码下超参数 $\eta$ 不同时的 mAP

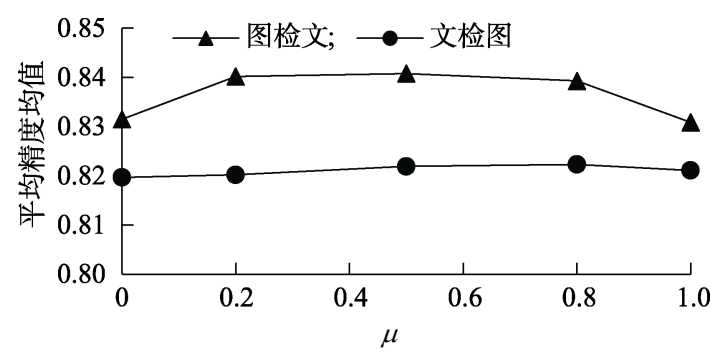

图 464 位码下超参数 $\mu$ 不同时的 $\mathrm{mAP}$

\section{4 结 语}

本文提出了一种有效的基于对抗投影学习的
跨模态哈希方法 APLH．该方法考虑保持语义信息 在特征学习中的重要性, 将跨模态投影匹配约束 引人特征学习模块, 使特征投影匹配分布和标签 投影匹配分布趋于一致，从而关联具有高相关性 的跨模态数据. 此外, 本文的深度对抗网络模型在 学习哈希函数时, 使用了加权余弦三元组损失函 数以及基于线性分类器的离散哈希方法, 在哈希 码中保证了模态间的语义一致性，同时大大减小 了量化损失. 在 3 个公开跨模态数据集上与多个先 进的跨模态哈希方法进行实验比较, 为验证 CMPM 约束的有效性进行了消融实验, 为研究某 些参数对实验结果的影响和敏感性进行了参数分析 实验. 结果表明，本文方法在检索性能方面具有较 好的优越性, 对关键超参数变化具有鲁棒性.

\section{参考文献(References):}

[1] Lin Z J, Ding G G, Hu M Q, et al. Semantics-preserving Hashing for cross-view retrieval[C] //Proceedings of the IEEE Conference on Computer Vision and Pattern Recognition. Los Alamitos: IEEE Computer Society Press, 2015: 3864-3872

[2] Ding G G, Guo Y C, Zhou J L. Collective matrix factorization Hashing for multimodal data[C] //Proceedings of the IEEE Conference on Computer Vision and Pattern Recognition. Los Alamitos: IEEE Computer Society Press, 2014: 2083-2090

[3] Zhang D Q, Li W J. Large-scale supervised multimodal Hashing with semantic correlation maximization[C] //Proceedings of the AAAI Conference on Artificial Intelligence. Palo Alto: AAAI Press, 2014: 2177-2183

[4] Wang D, Gao X B, Wang X M, et al. Semantic topic multi-modal Hashing for cross-media retrieval[C] // roceedings of the International Joint Conference on Artificial Intelligence. Palo Alto: AAAI Press, 2015: 3890-3896

[5] Krizhevsky A, Sutskever I, Hinton G E, et al. ImageNet classification with deep convolutional neural networks[C] //Proceedings of the 25th International Conference on Neural Information Processing Systems. New York: Curran Associates Inc., 2012: 1097-1105

[6] Bai C, Huang L, Pan X, et al. Optimization of deep convolutional neural network for large scale image retrieval[J]. Neurocomputing, 2018, 303: 60-67

[7] Bai C, Chen J N, Huang L, et al. Saliency-based multi-feature modeling for semantic image retrieval[J]. Journal of Visual Communication and Image Representation, 2018, 50: 199-204

[8] Lu J W, Liong V E, Zhou J. Deep Hashing for scalable image search[J]. IEEE Transactions on Image Processing, 2017, 26(5): 2352-2367

[9] Müler H, Unay D. Retrieval from and understanding of large-scale multi-modal medical datasets: a review[J]. IEEE Transactions on Multimedia, 2017, 19(9): 2093-2104

[10] Zhang J L, Liu P, Zhang F, et al. CloudNet: ground-based cloud classification with deep convolutional neural network[J]. Geophysical Research Letters, 2018, 45(16): 8665-8672 
[11] Zhang J, Peng Y X, Yuan M K. SCH-GAN: semi-supervised cross-modal Hashing by generative adversarial network[J]. IEEE Transactions on Cybernetics, 2020, 50(2): 489-502

[12] Zhang Y, Lu H C. Deep cross-modal projection learning for image-text matching[C] //Proceedings of the European Conference on Computer Vision. Heidelberg: Springer, 2018: 707-723

[13] Zhang J, Peng Y X, Yuan M K. Unsupervised generative adversarial cross-modal Hashing[C] //Proceedings of the AAAI Conference on Artificial Intelligence. Palo Alto: AAAI Press, 2018: 539-546

[14] Wu G S, Lin Z J, Han J G, et al. Unsupervised deep Hashing via binary latent factor models for large-scale cross-modal retrieval[C] //Proceedings of the International Joint Conference on Artificial Intelligence. Palo Alto: AAAI Press, 2018: 2854-2860

[15] Jiang Q Y, Li W J. Deep cross-modal Hashing[C] //Proceedings of the IEEE Conference on Computer Vision and Pattern Recognition. Los Alamitos: IEEE Computer Society Press, 2017: 3270-3278

[16] Li C, Deng C, Li N, et al. Self-supervised adversarial Hashing networks for cross-modal retrieval[C] //Proceedings of the IEEE Conference on Computer Vision and Pattern Recognition. Los Alamitos: IEEE Computer Society Press, 2018: 4242-4251

[17] $\mathrm{Gu}$ W, Gu X Y, Gu J Z, et al. Adversary guided asymmetric Hashing for cross-modal retrieval[C] //Proceedings of the ACM International Conference on Multimedia Retrieval. New York:
ACM Press, 2019: 159-167

[18] Chatfield K, Simonyan K, Vedaldi A, et al. Return of the devil in the details: delving deep into convolutional nets[C] //Proceedings of the British Machine Vision Conference. Cambridge: British Machine Vision Association, 2014: 1-12

[19] Gui J, Liu T L, Sun Z N, et al. Fast supervised discrete Hashing[J]. IEEE Transactions on Pattern Analysis and Machine Intelligence, 2018, 40(2): 490-496

[20] Huiskes M J, Lew M S. The MIR Flickr retrieval evaluation[C] //Proceedings of the ACM International Conference on Multimedia Information Retrieval. New York: ACM Press, 2008: 39-43

[21] Chua T S, Tang J H, Hong R C, et al. NUS-WIDE: a real-world web image database from National University of Singapore[C] //Proceedings of the ACM International Conference on Image and Video Retrieval. New York: ACM Press, 2009: 368-375

[22] Costa Pereira J, Coviello E, Doyle G, et al. On the role of correlation and abstraction in cross-modal multimedia retrieval[J]. IEEE Transactions on Pattern Analysis and Machine Intelligence, 2014, 36(3): 521-535

[23] Yang E K, Deng C, Liu W, et al. Pairwise relationship guided deep Hashing for cross-modal retrieval[C] //Proceedings of the AAAI Conference on Artificial Intelligence. Palo Alto: AAAI Press, 2017: 1618-1625

[24] Chen T Y, Zhang L, Zhang S C, et al. Extensible cross-modal Hashing[C] //Proceedings of the International Joint Conference on Artificial Intelligence. Palo Alto: AAAI Press, 2019: 21092115 